\title{
The effects of attention on age-related relational memory deficits: Evidence from a novel attentional manipulation
}

\author{
So-Yeon Kim ${ }^{1}$ and Kelly S. Giovanello ${ }^{1,2}$ \\ ${ }^{1}$ Department of Psychology, University of North Carolina at Chapel Hill \\ ${ }^{2}$ Biomedical Research Imaging Center, University of North Carolina at Chapel Hill
}

\section{Abstract}

\begin{abstract}
Healthy aging is often accompanied by episodic memory decline. Prior studies have consistently demonstrated that older adults show disproportionate deficits in relational memory (RM) relative to item memory (IM). Despite rich evidence of an age-related RM deficit, the source of this deficit remains unspecified. One of the most widely investigated factors of age-related RM impairment is a reduction in attentional resources. However, no prior studies have demonstrated that reduced attentional resources are the critical source of age-related RM deficits. Here, we utilized qualitatively different attention tasks, and tested whether reduced attention for relational processing underlies the RM deficit observed in aging. In Experiment 1, we imposed either itemdetection or relation-detection attention tasks on young adults during episodic memory encoding, and found that only the concurrent attention task involving relational processing disproportionately impaired RM performance in young adults. Moreover, by ruling out the possible confound of task-difficulty on the disproportionate RM impairment, we further demonstrated that reduced relational attention is a key factor for the age-related RM deficit. In Experiment 2, we replicated the results from Experiment 1 using different materials of stimuli and found that the effect of relational attention on RM is material-general. The results of Experiment 2 also showed that reducing attentional resources for relational processing in young adults strikingly equated their RM performance to that of older adults. Thus, the current study documents the first evidence that reduced attentional resources for relational processing are a critical factor for the relational memory impairment observed in aging.
\end{abstract}

\section{Keywords}

Aging; Episodic memory; Attention; Association

\begin{abstract}
Why do older adults often forget about everyday episodes? As people age, not only their physical health, but also some cognitive abilities decline. Among the cognitive functions affected by aging, episodic memory decline is a well established finding in the field of cognitive aging. Episodic memory refers to memory of events and experiences that have happened in the individuals' personal past. This memory system involves the conscious encoding and retrieval of contextually-specific information, such as memory for perceptual,
\end{abstract}

Corresponding author: So-Yeon Kim, Ph.D., Now at Center for Mind and Brain, University of California at Davis, 202 Cousteau Place Davis, CA 95618, Telephone: (530) 297-4651, Fax: (530) 297-4400, soykim@ucdavis.edu.

Publisher's Disclaimer: The following manuscript is the final accepted manuscript. It has not been subjected to the final copyediting, fact-checking, and proofreading required for formal publication. It is not the definitive, publisher-authenticated version. The American Psychological Association and its Council of Editors disclaim any responsibility or liabilities for errors or omissions of this manuscript version, any version derived from this manuscript by NIH, or other third parties. The published version is available at www.apa.org/pubs/journals/pag. 
conceptual, and affective components that are placed within an ongoing context of personal events (Tulving, 1983). A rich body of literature suggests that older adults have a disproportionate deficit in episodic memory relative to other types of memory due to their problems in remembering contextual details (e.g., Burke \& Light, 1981). Age-related impairments in contextual details have been found in memory for location (Glisky, Rubin, \&Davidson, 2001), temporal order (Fabiani \& Friedman, 1997; Cabeza, Anderson, Houl, Mangels \& Nyberg, 2000), and perceptual attributes of the information (Naveh-Benjamine \& Craik, 1996; Pilotti, Mead \& Gallo, 2003).

Remembering contextual information largely relies on successful binding of an event with its context. In accordance with this idea, Chalfonte and Johnson (1996) generalized the age difference in memory for contextual information to a binding deficit, by suggesting that the ability to integrate pieces of information into complex memories may be diminished with aging. To test their hypothesis, the researchers manipulated study instruction, by asking one set of participants to study only a single stimulus (a simple line drawing) or only contextual information (color or location of the drawing), while instructing the other group to study combined stimuli (i.e., either drawing with its location or drawing with its color). During the test phase, participants were presented with three types of stimuli. Some drawings were presented in the same color or location as at study (i.e., intact), and other drawings were presented in a new color or location (i.e., recombined). Unstudied drawings (i.e., new) were also included at test. Participants were asked to discriminate "intact" stimuli among these three types of stimuli. The researchers found that older adults' memory for individual object or contextual information (e.g., colors or locations) was just as good as that of young adults. However, the older adults were less able to remember object-color or object-location pairs relative to young adults. This finding suggests that older adults have a specific difficulty in associating or binding together distinct pieces of information, rather than a general deficit in encoding new information or new context.

Since the findings of Chalfonte and Johnson (1996), there has been an accumulation of evidence supporting the claim that older adults experience an inability to bind features together into relatively complex memories (Bayen, Phelps, \& Spaniol, 2000; Mitchell, Johnson, Raye, \& D'Esposito, 2000; Naveh-Benjamin, 2000). Critically, Naveh-Benjamin (2000) expanded the scope of the feature-binding notion in age-related memory deficits by proposing the Associative-Deficit Hypothesis (ADH) which focuses on the differential effects of aging on item vs. relational memory. To this end, he demonstrated that older adults have disproportionate deficits in relational memory (i.e., memory for associations among stimuli, $R M$ ) compared to their relatively intact item memory (i.e., memory for individual items, $I M)$. Specifically, Naveh-Benjamin (2000) employed a method originated by Humphreys (1976) to demonstrate RM deficits in older adults. The method was developed to assess memory for item and associative information separately (Humphreys, 1976). In this method, participants study a list of pairs of items (e.g., A-B, C-D). During the test phase for IM, participants receive some of the original items paired with some new items, and are asked to recognize the items that they saw in the study phase (e.g., detect A in A-F). For testing RM, participants also receive pairs of items, but pairs either can intact pairs (e.g., A-B) or recombined pairs which include items that were presented during the study phase, but not together (e.g., A-C). In the RM test, participants must discriminate between intact and recombined pairs. Using such procedures, Naveh-Benjamin (2000) tested the ADH in a series of experiments and found a disproportionate RM deficit in older adults for their memory for both inter- and intra-item relations, relative to memory for items alone. In particular, he tested memory for inter-item associations using unrelated word-nonword pairs and unrelated word-word pairs, and examined intra-item associations using word-font pairs. In a set of experiments, the author demonstrated that memory for single items (words or fonts) was comparable between older and young adults, whereas memory for 
conjunctions of items was disproportionately deficient in older adults. Since the initial proposal of the ADH, a growing body of research has provided evidence for age-related RM deficits using various materials, such as object pairs (Naveh-Benjamin, Hussain, Guez, \& Bar-On, 2003), face-name pairings (Naveh-Benjamin, Guez, Kilb, \& Reedy, 2004a; Troyer, Hafliger, Cadieux, \& Craik, 2006), face pairs (Bastin \& Van der Linden, 2006; Rhodes, Castel, \& Jacoby, 2008), and pairs of acting agents and actions (Old \& Naveh-Benjamin, 2008).

Despite the rich evidence for a disproportionate RM deficit in aging, the source of this deficit is still unclear. Several studies have focused upon the relationship between reduced attentional resources and the RM deficit in aging. In fact, Craik and colleagues (Craik, 1983; Craik \& Byrd, 1982) have proposed the common mechanism view which considers reduced attentional resources as a major cause of episodic memory problems in older adults. Craik and colleagues (1996) have shown that dividing attention at the time of encoding greatly reduces subsequent cued recall of unrelated noun pairs, and this impaired performance may be attributed to a failure to establish the adequate relational linkage between the component items. Subsequent research supported this view by showing that divided attention (DA) at encoding worsened memory for contextual information (Troyer \& Craik, 2000; Troyer, Winocur, Craik, \& Moscovitch, 1999). Moreover, Anderson et al. (1998) showed that DA at encoding disrupted memory performance of both young and older adults in free recall, cued recall, and recognition tasks. Additionally, using various secondary tasks and episodic memory tasks, Fernandes and Moscovitch (2000) found that dividing one's attention at encoding disturbed performance on episodic memory tasks.

Although previous research has consistently provided evidence for a significant effect of reduced attentional resources on episodic memory performance, it is still unclear whether a lack of attentional resources also mediates the RM deficits observed in older adults. To test the effects of attention on RM, researchers have given young adult participants a secondary task, while they encoded memory lists, and subsequently tested whether the participants showed disproportionate RM deficits under divided attention (DA), relative to full attention (FA) condition (Castel \& Craik, 2003; Naveh-Benjamin et al., 2003, 2004; Kilb \& NavehBenjamin, 2007).

For example, Naveh-Benjamin and colleagues (2003) provided a group of young adults with a secondary digit-monitoring task while they encoded pairs of object pictures (DA condition). Another young adult group received the study list without any concurrent tasks (FA condition). The authors found that the young adults under DA condition showed reduced accuracy, indicative of a general decline in memory performance. Nonetheless, unlike the pattern of the older adults' RM deficits, the secondary task affected memory performance for item and association proportionately. This proportionate effect of reduced attention in young adults on IM and RM has been consistently found in later studies with the DA paradigm using different mnemonic materials and different attention tasks (e.g., visual or auditory detection tasks) (Naveh-Benjamin, Guez, \& Shulman, 2004b; Kilb \& NavehBenjamin, 2007; Craik, Luo, \& Sakuta, in press). Furthermore, Castel and Craik (2003) also demonstrated that reducing attentional resources in young adults did not equate their RM performance to that of older adults. Specifically, they observed a disproportionate RM deficit in young adults under the DA condition, but the pattern of the deficit in young and older adults was different; young adults' RM impairments were due to their reduced hit rates, whereas the age-related RM deficit was due to increased false alarm rates. Overall, previous researchers concluded that a reduction in attention may not be a critical factor for the age-related RM deficit. 
Despite the previous conclusion, here we propose an alternative account in which reductions in a specific type of attentional resource plays a critical role in the age-related RM deficit. It has been demonstrated that attention involves different types of processing (selective, inhibitory, or sustained attention) and distinct types of domains (visual, verbal, or spatial attention) (Navon \& Gopher, 1979). Furthermore, it has been well-demonstrated that attention interacts with other memory systems, such as working memory (WM), in a resource-dependent manner (e.g., Kim, Kim, \& Chun, 2005). Specifically, Kim and colleagues (2005) demonstrated that the performance on an attention task can be significantly improved or disturbed depending on the relation between resources required for concurrent WM and resources required for the attention tasks. Using a variety of attention and WM tasks, the authors showed that WM load significantly disturbs performance in an attention task when a concurrent WM task utilizes the same type of cognitive resource (e.g., spatial or verbal) as target processing in the attention task, whereas a WM task can in fact improve selective attention processing when the type of WM load overlaps with the distractor processing.

Despite evidence that attention and other cognitive functions interact in a resourcedependent manner, previous studies testing the effect of attention on RM have used attention tasks which tag general attentional resources rather than attentional resources that may be required for RM. In the current study, we hypothesize that older adults experience a reduction in the ability to allocate attention to relational aspects of stimuli, which in turn causes the age-related RM deficit. Here, we labeled this attentional process as relational attention. To test our hypothesis, we divided participants into separate attention groups (i.e., a full attention and different divided attention groups), and tested their item- and association-recognition abilities under different attentional loads at encoding. Critically, we utilized attention tasks which specifically involve relational processing in order to reduce participants' resources for relational attention. Secondary tasks which require little or no relational processing were also used. Using such tasks, two experiments examined whether a lack of relational attention processing may account for the age-related RM deficit.

\section{Experiment 1}

\section{Methods}

Participants-Thirty six undergraduate students from the University of North Carolina at Chapel Hill (UNC-CH) participated and received course credits (Age: 17-25, Mean=19.7, $\mathrm{SD}=1.73$; Female: 22). Participants were randomly assigned to one of three attention groups: Full attention (FA), Divided attention with Item processing (DA-I), and Divided attention with Relational processing (DA-R). All participants were native English speakers and none of the participants was aware of the purpose of the current experiment. The experimental procedure was approved by the Institutional Review Board at UNC-CH.

Stimuli-There were three attention groups in this experiment and a total 96 common words with less than four-syllables were used for two memory tests (i.e., IM and RM) in each of the attention condition. For the two divided attention (DA) conditions (i.e., DA-I and DA-R), 128 face stimuli were used during the encoding phase, which were selected from the PAL face database (Minear \& Park, 2004; 64 females and 64 males; age 30-90). Different gendered faces (age gap: 5-55 years) were paired on each trial of the encoding phase in both DA conditions. For the full attention (FA) condition, scrambled images of the face stimuli were used to balance the amount of visual inputs during the encoding phase across all attention conditions. After the encoding phase, all participants completed both item and relational memory tests and the order of the tests was counterbalanced across all participants. Each test phase consisted of three types of pairs: word pairs consisted of previously seen pairs (i.e., intact), pairs of two words presented in the encoding phase but 
not together (i.e., recombined), and pairs with two new words (i.e., new). All stimuli were counterbalanced across participants using a Latin square design. All experiments were presented on an Apple iBookG4 using a program, MacStim (Darby, 2006).

Procedures-Each encoding block consisted of 32 unrelated word pairs. Each pair was presented for 4 seconds. To avoid possible floor effects (see Anderson et al., 1998), intentional encoding was used in which participants were instructed to memorize individual words and word pairs to prepare for the later recognition tests. Participants in the DA with item processing (DA-I) group were asked to detect the location of the male face between two faces during each memory encoding trial, whereas participants in the DA with relational processing (DA-R) group were asked to compare the ages of two faces and to detect the location of the older face. Face pairs were presented for 2 seconds in both conditions; thus, each word pair was accompanied by two different face pairs. The word pairs switched their locations every 2 seconds to produce the equivalent visual changes as the face pairs and to avoid possible involuntary attentional capture to the face pair changes. Participants in both DA groups were instructed to pay equal attention to word and face pairs. Participants in the FA group were presented with a word pair and a pair of scrambled images during the encoding phase. The images were presented in the same location as the face stimuli in the DA conditions to equate the perceptual load between full and divided attention conditions. However, participants were instructed to ignore the scrambled images during encoding. Both images and words switched their locations every 2 seconds to mimic the trials in the DA groups. Figure 1 depicts the procedure for the encoding blocks in Experiment 1.

Each participant received one encoding phase, and item memory (IM) and relational memory (RM) tests followed after an interpolate activity of 60 seconds (subtracting 3 continuously from 1000). In both IM and RM blocks, 24 word pairs were presented and each pair was presented for 4 seconds; 8 intact, 8 recombined, and 8 new pairs. In the IM test, participants were asked to decide whether they saw both words in the encoding phase and to press either "YES" (for the intact and recombined pairs) or "NO" button (for the new pairs). In the RM test, participants were instructed to determine whether they saw both words together during the study phase. Thus, the correct answer for intact pairs was "YES", while the correct answer for recombined or new pairs was "NO" in the RM test. The order of the tests was counterbalanced across all participants.

\section{Results}

Attention performance-Accuracy and reaction times (RT) for correct responses were averaged for each divided attention task, and the results are presented in Table 1A. Independent samples t-tests revealed significant differences in accuracy, $M D$ (mean difference $)=.20, t(22)=12.63, p<.05, r^{2}=.94$, as well as in RT, $M D=182 \mathrm{~ms}, t(22)=2.53, p<$. $05, r^{2}=.47$. That is, participants in DA-R group performed significantly worse in their attention task than participants in DA-I group. These results indicated that detecting "older faces" in the relational attention task was more difficult than detection of "male faces" in the item attention task.

Memory accuracy-Figure 2 shows the proportion of hits minus proportion of false alarm rates for the IM and RM tests for each group (c.f., Please see the first three sets of graphs). A 3 (Attention, between-subject factor) $\times 2$ (Memory, within-subject factor) mixed ANOVA revealed a significant main effect of Memory test, $F(1,33)=17.13, p<.05, \eta^{2}=.25$, and a significant main effect of Attention group, $F(2,33)=10.26, p<.05, \eta^{2}=.34$. The interaction between attention and memory tasks was also significant, $F(2,33)=8.63, p<.05, \eta^{2}=.26$, suggesting different effects for each attentional load on IM and RM tests. To test the locus of the interaction, post-hoc t-tests for each attention group were conducted. The results 
showed significantly worse performance in the DA-R group in the RM test relative to their performance in the IM test, $M D=.26, t(11)=5.36, p<.05, r^{2}=.85$. Such an effect of attentional load was not evident in either DA-I or FA group, suggesting that only the attentional load involving relational processing disproportionately affected performance on the RM test.

Retrieval latency-To test differences in the memory retrieval reaction times (RT), a 3 (Attention, between-subject factor) $\times 2$ (Memory, within-subject factor) mixed ANOVA was conducted on the retrieval latencies (Table 1B). The results showed no significant main effect of memory, $F(1,33)<1$, nor attention group, $F(2,33)<1$, on the retrieval latencies. Also, the interaction between the two factors was not significant, $F(2,33)=2.491, p>.05$. These results confirm that the findings from the memory accuracy data are not a consequence of speed-accuracy trade-off.

\section{Additional Attention Condition: Addressing an issue of the task difficulty}

The current experiment demonstrates that divided attention at encoding disproportionately affects young adults' RM performance when the attention task requires relational processing. However, different levels of performance in the two attentional tasks limits the conclusion that a specific type of attentional resources, namely relational attention, is the critical factor for the disproportionate RM impairments observed in the current study. Because the relational attention task was more difficult than the item attention task, it is plausible that the disproportionate effect of relational attention task is not due to the specific type of attention task imposed during encoding, but rather, due to larger attentional demands in the relational- than in the item-attention task. To address this question of task difficulty, we added an additional attention group, where participants performed a more difficult item attention task during encoding of memory stimuli (i.e., divided attention with item, harder group: DA-IH).

In the DA-IH condition, twelve additional undergraduate students from the UNC-CH were recruited (Age: 19-23, Mean=20.4, SD=1.38; Female=5). Participants in this group performed the same attention task as those in the DA-I group (i.e., detection of male faces), but the inter stimuli intervals (ISIs) of the face and word stimuli were reduced by half in order to increase the overall difficulty of the attention and memory tasks. That is, during the encoding phase, participants in this group were instructed to detect male faces every one second and each word pair switched their location every one second. Following the encoding phase, all participants in the DA-IH group received the same IM and RM tests as those in other attention groups, and the order of the memory test was counterbalanced across all participants.

The results from the DA-IH group were analyzed with the previous results in this experiment. Since the ISIs in the attention tasks were different between the DA-IH group and the DA-I/DA-R groups (i.e., 1 vs. 2 seconds), only the accuracy data were analyzed for the three attention tasks. As predicted, the attention task in the DA-IH condition was more difficult than the attention task in the DA-I condition. Specifically, a one-way ANOVA with accuracy data in all three divided attention tasks showed significant differences in accuracy among three groups, $F(2,33)=19.38, p<.05, \eta^{2}=.54$. Post-hoc t-tests revealed that attention accuracy in the DA-IH group was significantly lower than that of DA-I group, $M D=.19$, $t(22)=4.45, p<.05, r^{2}=.69$. The level of difficulty of the item attention task in the DA-IH group was not, however, significantly different from that of the relational attention task in the DA-R group, $M D=-.01, t(22)=-.182, p>.05$. That is, the task difficulty of the two attention tasks (i.e., harder item attention task vs. relational attention task) was comparable from each other. 
Despite the increased difficulty in the item attention tasks in the DA-IH condition, the pattern of the results in the memory tests was comparable with that of the DA-I condition (Figure 2). Specifically, a 4 (Attention group) $\times 2$ (Memory test) ANOVA showed significant main effects of Memory test and Attention group, $F(1,44)=16.143, p<.05, \eta^{2}=$. 19 , and $F(3,44)=13.92, p<.05, \eta^{2}=.49$, respectively, as well as a significant interaction between two factors, $F(3,44)=7.77, p<.05, \eta^{2}=.28$. Importantly, the significant interaction was resulted from disproportionately lower RM performance in the DA-R group. Despite the equivalent level of difficulty in attention task in the DA-IH group compared to that of DA-R group, the harder item attention task affected the IM and RM performance proportionately, $M D=.02, t(11)=.49, p>.05$. That is, the harder attention task, which requireds little or no relational processing, significantly worsened both IM and RM performance compared to the easier version of the same attention task, but the degree of the impairment in two memory tests were proportionate. These results support the notion that a specific type of attentional process, namely relational attention, is an important factor for the disproportionate RM impairment observed in aging.

\section{Discussion}

Unlike previous studies of divided attention and RM (e.g., Castel \& Craik, 2003; NavehBenjamin et al., 2003; 2004; 2007), the current experiment manipulated the type of attentional resource required for the concurrent attention tasks during memory encoding. Using different types of attention tasks imposed during encoding, the present study tested the hypothesis that reducing resources for relational attention would disproportionately impair young adult's performance on a RM test. The results supported our hypothesis that imposing an attention task which involved relational processing would disproportionately affect RM, compared to IM, performance. Furthermore, a different attention task, which involved minimal relational processing, impaired both item and relational memory performance proportionately. Notably, the different effects of two attention tasks on IM and RM tests were not due to different levels difficulty. More specifically, the more difficult item attention task affected both IM and RM tests proportionately unlike the relational attention task which impaired RM performance disproportionately. Thus, the current study provides the first evidence that a reduction in attentional resources specific to relational processing may be a crucial factor for the disproportionate RM deficits observed in aging.

Although the current experiment demonstrated that resources for relational attention significantly interact with performance on a RM test, several components of the design merit follow-up. First, it is worthwhile to test whether the interaction between relational attention and RM can be manipulated when different types of materials are used in the attention and memory tasks. Previous studies examining the interaction between attention and other cognitive processes (e.g., working memory) suggest that the interaction between various cognitive systems is different depending on the type of materials used in each cognitive task (Kim, Kim, \& Chun, 2005; Oh \& Kim, 2004, Woodman \& Luck, 2004). In the current experiment, resources within the visual domain were used in the attention tasks (i.e., pictures of faces), while resources within the verbal domain were utilized in the memory tasks (i.e., word pairs). Therefore, it is of interest to examine whether the effect of relational attention would be increased or decreased when the same domain is used for both in attention and memory tasks. Experiment 2 was thus designed in part to address this question by examining whether or not the effect of the relational and/or item attention tasks on memory performance would be altered due to changes in materials in the attention tasks.

Second, the goal of the current study was to test whether the age-related RM deficit would be observed in young adults when their cognitive resources for relational attention processing were diminished. In Experiment 1, we found that young adults under DA with relational processing showed a disproportionate impairment in RM relative to IM. However, 
this result does not provide direct evidence that the pattern of the RM deficit in those young adults was equivalent to the older adult's RM deficit because older adults were not tested in this experiment. Hence, in Experiment 2, we recruited healthy older adults and examined their performance in the same IM and RM tests as for young adults. Critically, the pattern of the RM deficit in older adults was directly compared to that of young adults under DA with relational attention in order to investigate the importance of relational attention resources for relational memory.

\section{Experiment 2}

\section{Methods}

Participants-Forty eight undergraduate students (Age: 18-28, Mean=19.7, SD=1.89; Female: 33) from the UNC-CH, who did not participate in Experiment 1, participated for course credit. Young adults were randomly assigned to one of four conditions (12 young adults per group): FA, DA-I, DA-R, and DA-IH. In addition, 12 older adults (Age: 70-81, Mean=74.6, SD=3.20; Female: 5) were recruited from the local community and were paid $\$ 10$ per hour. Older adults participated in the FA condition only.

Stimuli-The word stimuli in the two memory tests were identical to those used in Experiment 1. However, for the secondary tasks, number stimuli (from one to ten) were used instead of pictorial stimuli, and all numbers were written in English alphabet (e.g., "THREE"). For the DA conditions, an odd and an even number were randomly paired. In the FA condition, meaningless strings of Xs and Ys were presented in the same location as the number stimuli in the DA conditions to balance the amount of visual inputs across all conditions.

Procedures-The procedures in Experiment 2 were identical to those in Experiment 1 with a couple of exceptions (Figure 3). Young adults in Experiment 2 were randomly assigned either to the FA or to one of the three DA conditions (DA-I, DA-R, or DA-IH). During encoding, participants in the DA-I group were asked to detect an odd number and individuals in the DA-R condition were asked to compare two numbers and to detect a numerically larger one between the two numbers. Thus, the attention task in the DA-R group required relational attention processing, whereas the attention task in the DA-I group did not. In addition to the two DA conditions, a more difficult item-based attention task condition (DA-IH) was included to rule out the effects of overall task difficulty on RM performance. Participants in the DA-IH group performed the same attention task as those in the DA-I group during encoding, but the presentation rate for stimuli in the attention task was accelerated. That is, each number pair was presented for 1 second instead of 2 seconds. Thus, participants in this group had to respond to four different number stimuli while encoding one word pair. Participants in the DA conditions were instructed to pay equal attention to both word and number pairs. Finally, participants in the FA condition were presented with a number pair and a meaningless string pair (strings of Xs and Ys) in each trial of memory encoding, and instructed to memorize the word stimuli ignoring the strings. Like previous studies on divided attention and RM deficits (e.g., Naveh-Benjamin et al., 2003; 2004), older adults were assigned to the FA group only to verify age-related RM deficits in the current experiment. After the study phase, all participants received the same delay activity, as well as the same IM and RM tests as Experiment 1.

\section{Results}

Attention performance-To test whether the level of difficulty was different among three attention groups, a one-way ANOVA was conducted for the accuracy data from the three divided attention groups. The result showed significant differences in accuracy, 
$F(2,33)=32.69, p<.05, \eta^{2}=.66$. Post-hoc t-tests revealed that attention accuracy in the DA-IH group was significantly lower than that of DA-I and of DA-R groups, $M D=.25, t(22)=7.28$, $p<.05, r^{2}=.84$, and $M D=.22, t(22)=5.84, p<.05, r^{2}=.78$, respectively. Unlike the results in Experiment 1 , accuracies from DA-I and DA-R groups were not significantly different from each other, $M D=.04, t(22)=1.21, p>.05$. Since the presentation rate in the DA-IH group was different from those in the DA-I and DA-R groups (i.e., 1 vs. 2 seconds), a comparison of reaction time (RT) data between DA-IH group and other two attention groups was not meaningful. Thus, only an independent samples t-test was conducted for RT data from DA-I and DA-R groups. As in the case of accuracy, the RT results from the two attention groups were not significantly different, $M D=-5, t(22)=-.08, p>.05$. In sum, these results confirmed that the attention task under DA-IH group was the hardest among three attention tasks, and the level of difficulty in attention tasks under DA-I and DA-R groups was equivalent in Experiment 2. The accuracy and RT data are listed in Table 2A.

Memory accuracy-Figure 4 shows the proportion of hits minus proportion of false alarm rates for each memory test for each group. A 5 (Attention group, between-subject factor) $\times 2$ (Memory, within-subject factor) mixed ANOVA revealed significant main effects of Attention group and Memory task, $F(4,55)=12.22, p<.05, \eta^{2}=.47$, and $F(1,55)=12.15, p<.05$, $\eta^{2}=.14$, respectively. The interaction between the two factors was also significant, $F(4,55)=4.29, p<.05, \eta^{2}=.20$, indicating that participants' performance in each memory task was different depending on the attention group. To address specific hypotheses to be tested in this experiment, we conducted additional ANOVAs for each hypothesis. First, to test for an age-related RM deficit, a $2 \times 2$ mixed ANOVA was carried out to compare young and older adults under FA conditions. The results revealed a significant main effect of Age group, $F(1,22)=16.16, p<.05, \eta^{2}=.42$, indicating significantly worse accuracy in older adults than young adults under FA. A main effect of Memory test was also significant, $F(1,22)=7.04, p<.05, \eta^{2}=.20$, indicating poorer performance in RM than IM. This main effect of Memory test was due to a reduction in RM in older adults, as revealed by the significant interaction between Memory test and Age group, $F(1,22)=7.04, p<.05, \eta^{2}=.20$. Specifically, post-hoc comparisons showed no age differences in IM, $M D=.10, t(22)=1.69$, $p>.05$, but there was a significant difference between young adults and older adults in RM, $M D=.34, t(22)=4.28, p<.05, r^{2}=.67$. Finally, separate paired t-test for each age group revealed significant differences between the two memory tests in the older adult group only, $M D=.24, t(11)=3.67, p<.05, r^{2}=.74$, confirming the disproportionate RM deficit in older adults.

Next, to test the effect of different attentional loads on memory performance, a $4 \times 2$ mixed ANOVA was performed including young adults only. This test revealed a significant main effect of Attention group, $F(3,44)=15.27, p<.05, \eta^{2}=.51$, indicating that participants in the DA groups performed worse in both of memory tests. Interestingly, the interaction between Attention group and Memory test was also significant, $F(3,44)=3.19, p<.05, \eta^{2}=.17$. The results from the post-hoc t-tests indicated that only the young adults under DA-R group showed significantly worse accuracy in RM test compared to their IM performance, $M D=$. $19, t(11)=3.14, p<.05, r^{2}=.69$. Importantly, the disproportionate effect of attention task on IM and RM in young adults in the DA-R group was strikingly similar to the disproportionate RM deficits in older adults. Overall, these results indicate that reducing resources for relational attention disproportionately impaired RM performance in young adults, while attentional loads which involved minimal relational processing proportionately affected performance in IM and RM tasks. Furthermore, the effect of relational attention was not due to the overall task difficulty, as revealed by proportionate memory impairments in IM and $\mathrm{RM}$ tests in young adults under the most difficult condition (i.e., in the DA-IH group, $M D=$ $-.04, t(11)=-.64, p>.05)$. 
Retrieval latency-The group means for retrieval latency in Experiment 2 are displayed in Table 2B. A 5 (Attention group, between-subject factor) $\times 2$ (Memory, within-subject factor) mixed ANOVA was conducted to test differences in the memory retrieval latencies in each group. The results revealed neither a significant main effect of memory, $F(1$, $55)=2.18, p>.05$, nor an interaction between the two factors, $F(4,55)=2.31, p>.05$, indicating no speed-accuracy trade-off in the memory data. However, the main effect of group was significant, $F(4,55)=7.76, p<.05, \eta^{2}=.36$. Post-hoc testing showed significantly slower retrieval response times (RTs) in older adults compared to those in young adults under each attention group; $M D=450 \mathrm{~ms}, 287 \mathrm{~ms}, 316 \mathrm{~ms}$, and $486 \mathrm{~ms}$ for young adults under FA, DAI, DA-R, and DA-IH group respectively, all $p s<.05$. Retrieval latencies for young adults under the different attention groups were not significantly different from each other. These results indicate that older adults took longer to respond than young adults for both memory tasks.

Although older adults were generally slower to retrieve information compared to young adults, older adults may experience more difficulty in retrieving associative information and, in turn, take longer time to respond in a RM test compared to an IM test. To investigate this hypothesis, a two way ANOVA with age groups (young and older adults under FA) and memory tests (IM and RM) as factors was carried out. The results verified the hypothesis, showing a significant interaction between age group and memory test for the retrieval RTs, $F(1,22)=4.504, p<.05, \eta^{2}=.13$. Post-hoc t-tests confirmed that only the older adults showed significantly slower RTs in the RM than in the IM test, $M D=196 \mathrm{~ms}, t(11)=4.480, p<.05$, $r^{2}=80$. Interestingly, separate two way ANOVAs comparing older adults to young adults under each attention group (DA-I, DA-R, DA-IH) resulted in a significant interaction between age group and memory test, with the exception of the ANOVA with older adults under FA and young adults under DA-R condition, $F(1,22)=1.981, p>.05$. These results thus provide additional evidence for the similar pattern of RM deficits in older adults under full attention and young adults with reduced resources for relational attention during encoding.

\section{Discussion}

The results from Experiment 2 replicated the findings from Experiment 1 and confirmed the hypothesis that age-related RM deficits may be mediated by a reduction in attentional resources specific to relational processing. Furthermore, using different materials in the attention task, the results in Experiment 2 generalized the effect of relational attention on $\mathrm{RM}$ to different modalities. This result is consistent with previous findings which showed general RM deficits in older adults, regardless of the type of association, such as within- or between- domain associations (Bastin \& Van der Linden, 2006). For example, Bastin and Van der Linden (2006) compared older adults' recognition performance on face-face pairs (i.e., within-domain association) to their performance on recognition of face-spatial pairs (i.e., between-domain association) and found that the degree of older adults' impairment in RM was equal across different types of association (e.g., within- or between-domain). In other words, the authors demonstrated that an age-related RM deficit was a domain-free rather than a domain-specific deficit in terms of materials used in the memory tasks. Consistent with this notion, the current study demonstrated that the effect of relational attention on RM was also domain-general, similar to the effect of aging on RM.

In addition, the results from Experiment 2 rule out the possibility that the RM deficit in young adults under relational attention condition was mediated by an increased task difficulty in the attention task. Using a more difficult attention task involving minimal relational processing (i.e., DA-IH group), the current study showed that the more difficult item-attention task proportionately impaired item and relational memory performance in young adults, unlike the case of RM deficits observed in older adults. Furthermore, the level 
of difficulty for the item and relational attention tasks in Experiment 2 was not significantly different from each other, as revealed by the similar accuracy and reaction time data between the two attention tasks. In other words, detection of a numerically larger number between two numbers was not attentionally more demanding than detection of an odd number, yet the two tasks differed with regard to their processing. That is, only the larger number detection task involved relational processing and only the young adults who performed memory encoding with this attention task showed disproportionately impaired performance on the associative memory task, relative to the item memory task. Moreover, their performance on the RM test was remarkably similar to older adults' deficits on the RM test.

The strikingly similar pattern of performance between young adults under the relational attention condition and older adults under the full attention condition is worth noting. Previously, Castel and Craik (2003) found a disproportionate RM deficit in young adults whose attention was divided during memory encoding. However, the authors indicated that the pattern of the deficit in young adults under DA condition was different from that of older adults with full attention. Specifically, while older adults' RM deficit was due to the increased false alarm rate for recombined pairs, the RM impairment in young adults under DA condition resulted from a decreased hit rate for intact pairs. Furthermore, the size of the deficit was larger in older adults compared to young adults under the DA condition. Unlike the findings by Castel and Craik (2003), the current study demonstrated that reducing a specific type of resource in young adults during memory encoding can in fact equate young adults' RM performance to that of older adults. That is, the disproportionately greater decrease in RM performance, relative to IM performance, in young adults under the DA-R condition resulted not only from a decreased hit rate for intact pairs, but also from increased false alarm rate for recombined pairs (see Table 3). Critically, this pattern of performance was also observed in older adults under the full attention condition, with an equivalent magnitude of impairment in young adults under the relational attention condition. Thus, the current study provides evidence that a reduction in attentional resources specific to relational processing is a crucial factor for the RM deficits observed in aging.

\section{General Discussion}

In two experiments, we demonstrated that reduced attention to relational aspects of information plays a critical role in the age-related RM deficit. Experiment 1 showed that a reduction in relational attention at encoding disproportionately impaired young adults' performance on a RM test relative to an IM test. Furthermore, we demonstrated that the disproportionate effect of relational attention on RM was not due to the overall task difficulty, as the more difficult attention task with minimal relational processing proportionally impaired IM and RM in young adults. Experiment 2 further demonstrated that reduced relational attention in young adults equated their RM performance to that of older adults under full attention conditions. Finally, reductions in relational attention affected RM performance in a modality-general manner: the effect of relational attention task with visual stimuli (Experiment 1) was similar to that of relational attention task involving verbal materials on a verbal RM task (Experiment 2). Overall, the current findings suggest that a reduction in relational attention plays a critical role in the age-related RM deficit.

Previously, Craik and colleagues proposed the common mechanism hypothesis, which posits that a major cause of episodic memory impairments in older adults is a reduction in attentional resources (Craik \& Byrd, 1982). This view has been supported by studies with DA and IM paradigms (Anderson, Craik, \& Naveh-Benjamin, 1998; Craik, Govoni, NavehBenjamin, \& Anderson, 1996), but not supported by studies with DA and RM paradigms. Specifically, previous studies demonstrated that the effect of age and the effect of DA are mediated, at least partially, by different mechanisms, by showing proportionate deficits in 
IM and RM in young adults with DA (e.g., Naveh-Benjamin et al., 2003), or by showing different patterns of RM impairments between young adults with DA and older adults with FA (Castel \& Craik, 2003). The current findings reconcile the conflict of the common mechanism view by indicating the importance to measure the effect of attentional reductions not only in quantitative aspects, but also in terms of qualitative features. More specifically, a quantitative reduction in attentional resources associated with aging may contribute to general impairments in IM and RM as shown in a linear decrease in performance in both memory tasks as a function of the difficulty of the attention task (i.e., DA-IH condition in both experiments). However, using qualitatively, but not quantitatively, different attentional loads, the current study also demonstrated that a reduction in attentional allocation to relational aspects of stimuli (i.e., relational attention) may account for age-related RM deficits.

One might question whether the current attentional manipulation is unique in terms of imposing attentional loads for relational processing. Previously, Castel and Craik (2003) used a digit monitoring task (i.e., detecting three consecutive odd digits) and found a disproportionate RM deficit in young adults under divided attention conditions (although the pattern of the deficit in young adults under DA condition was different from that of the older adults; see the discussion section of Experiment 2 for more details). Because the attention task used in Castel and Craik (2003) was different from the detection tasks used in other studies (e.g., Naveh-Benjamin et al., 2003, 2004, 2007), it is possible that the digit monitoring task played a role in disproportionately decreasing RM performance in young adults in the DA condition. However, the attention task used in Castel and Craik (2003) did not require relational processing. Instead, the digit monitoring task involved some degree of working memory processes to hold three successive odd digits. Thus, this attention task clearly requires more cognitive loads (selective attention and working memory) than a simple detection task, and this may lead to reductions in RM performance in young adults under the DA conditions. Nonetheless, as the authors discussed, the pattern of the RM deficit in young adults did not mimic the pattern shown by older adults. More recently, using a similar digit monitoring task, Craik, Luo, and Sakuta (in press) found proportionate memory declines for both item and relational memory tests when the load for digit monitoring was reduced to 2 digits from 3 digits. Unlike the aforementioned studies, we manipulated our attention task in terms of its processing (item vs. relational), and demonstrated that only the attention tasks which required relational processing disproportionately impaired young adults' RM performance. Critically, the pattern of performance in the DA with relational processing group was remarkably similar to the agerelated RM deficits, supporting our hypothesis that a lack of resources for relational attention plays a critical role in the age-related RM impairment.

Although this is the first study to demonstrate a significant effect of reduced relational attention on RM, two recent studies reported related findings. Firstly, Naveh-Benjamin and colleagues (2009) recently found that incidental learning of item and association eliminates the age-related deficit specific to associations. Using face and name pairs, the authors found that older adults reveal their typical RM deficits only under the intentional learning paradigm. In their conclusion, the authors emphasized the possible problem in older adults in which the older adults may have difficulty to initiate associative strategies when they intentionally memorize information. Given that the intentional learning requires more explicit attentional processing than the incidental learning, this recent finding also supports the idea that decreased attentional resources play an important role in age-related RM deficits. However, using different attentional manipulation, the current study directly tested the effects of different attentional resources on the age-related RM deficit. The data suggest that a reduction in the attentional resources for the relational processing is responsible for the disproportionate RM impairments observed in older adults. 
Additionally, Castel (2007) investigated the role of expertise in older adults' RM by testing whether expertise can benefit performance on a RM task which involved stimuli in an expertise-related domain. Using memory tasks involving expertise-related (i.e., number) and expertise-nonrelated domains (i.e., object), the author found that expertise reduced the agerelated RM deficit in the domain of the skill. Specifically, he replicated RM impairments in older adults in both object and number information, but the deficit disappeared for number targets in older adults with expertise in numbers. This recent finding highlights the importance of attentional allocation on relational aspects of stimuli, by showing that older adults who were trained or motivated to allocate sufficient attention to the relations between certain types of information (e.g., number and another stimulus) did not exhibit RM impairment for that information. The current findings also emphasize the importance of attentional resources allocated to the relational aspects of stimuli for RM. Whereas Castel showed reduced age-related RM deficits with larger allocation of attention to the stimuli, the current study demonstrated decreased young adults' RM performance with division of relational attention. Future studies integrating these two separate findings would elucidate the critical role of relational attention for the age-related RM deficit.

Although not tested in the current study, it would be beneficial to investigate the effect of reductions in relational attention during retrieval of RM in young adults. If older adults demonstrate deficits in RM due to their lack of resources for relational attention, it is possible that reductions in relational attention might play a role during RM retrieval as well. Previous researchers have in fact investigated the effect of divided attention (DA) at retrieval on RM, and demonstrated that DA at retrieval in young adults has little or no effect on memory performance, especially when recognition tasks are used (Craik, Govoni, NavehBenjamin, \& Anderson, 1996; Naveh-Benjamin, Craik, Guez, \& Dori, 1998; NavehBenjamin, Craik, Gavrilescu, \& Anderson, 2000; Anderson, Craik, \& Naveh-Benjamin, 1998). However, as in the case of DA at encoding, none of the studies manipulated the type of attention tasks imposed during retrieval. Although the current study demonstrated significant effects of DA at encoding with a relational attention task, in a future study, it would be informative to test whether reducing resources in relational attention during retrieval can also simulate the age-related RM deficit.

\section{Conclusion}

In summary, the current study contributes new knowledge to the cognitive aging literature. With respect to age-related RM deficits, the current findings provide evidence that a reduction in relational attention in older adults is a critical factor for the disproportionate RM deficits observed in older adults. Within the broader domain of episodic memory, these results suggest that a reduction in a specific aspect of attention can explain at least some aspects of age-related episodic memory decline. Understanding the nature and source of agerelated impairments in episodic memory is important for both theoretical and ecological reasons, since the ability to connect and associate separate pieces of information into contextual representation is essential for coherent memories of everyday events. With novel evidence for the source of RM deficits in older adults, the current study contributes to a more comprehensive picture of cognitive aging, especially in the domain of episodic memory decline.

\section{Acknowledgments}

We thank Jason Chavarria, Caleb Pardue, and Rebecca Lorenz for help with data collection and acknowledge NIAAG028774. 


\section{References}

Anderson ND, Craik FI, Naveh-Benjamin M. The attentional demands of encoding and retrieval in younger and older adults: 1. Evidence from divided attention costs. Psychology and Aging. 1998; 13(3):405-423. [PubMed: 9793117]

Bastin C, Van der Linden M. The effects of aging on the recognition of different types of associations. Experimental Aging Research. 2006; 32:61-77. [PubMed: 16293569]

Bayen UJ, Phelps MP, Spaniol J. Age-related differences in the use of contextual information in recognition memory: A global matching approach. Journals of Gerontology: Psychological Sciences. 2000; 55B:131-141.

Burke DM, Light LL. Memory and aging: The role of retrieval processes. Psychological Bulletin. 1981; 90:513-546. [PubMed: 7302054]

Cabeza R, Anderson ND, Houle S, Mangels JA, Nyberg L. Age-related differences in neural activity during item and temporal-order memory retrieval: A positron emission tomography study. Journal of Cognitive Neuroscience. 2000; 12:197-206. [PubMed: 10769316]

Castel AD. Aging and memory for numerical information: The role of specificity and expertise in associative memory. Journals of Gerontology Series B: Psychological Sciences and Social Sciences. 2007; 62(3):P194.

Castel AD, Craik FIM. The effects of aging and divided attention on memory for item and associative information. Psychology \& Aging. 2003; 18:873-885. [PubMed: 14692872]

Chalfonte BL, Johnson MK. Feature memory and binding in young and older adults. Memory \& Cognition. 1996; 24:403-416.

Craik FIM. On the Transfer of Information from Temporary to Permanent Memory. Philosophical Transactions of the Royal Society of London. Series B, Biological Sciences. 1983; 302:341-359.

Craik, FIM.; Byrd, M. Aging and cognitive deficits: The role of attentional resources. In: Craik, FIM.; Trehub, S., editors. Aging and cognitive processes. New York: Plenum Press; 1982. p. 199-211.

Craik FI, Govoni R, Naveh-Benjamin M, Anderson ND. The effects of divided attention on encoding and retrieval processes in human memory. Journal of experimental psychology. General. 1996; 125(2):159-180. [PubMed: 8683192]

Craik FI, Luo L, Sakuta Y. Effects of aging and divided attention on memory for item and their contexts. Psychology and Aging. in press.

Darby, D. MacStim. Melbourne: WhiteAnt Occasional Publishing; 2006.

Fabiani M, Friedman D. Dissociations between memory for temporal order and recognition memory in aging. Neuropsychologia. 1997; 35(2):129-141. [PubMed: 9025117]

Glisky EL, Rubin SR, Davidson PSR. Source memory in older adults: An encoding or retrieval problem? Journal of Experimental Psychology: Learning, Memory, and Cognition. 2001; 27:1131-1146.

Humphreys MS. Relational information and the context effect in recognition memory. Memory and Cognition. 1976; 4:221-232.

Kilb A, Naveh-Benjamin M. Paying attention to binding: Further studies assessing the role of reduced attentional resources in the associative deficit of older adults. Memory \& Cognition. 2007; 35(5): 1162-1174.

Kim S-Y, Kim M-S, Chun MM. Concurrent working memory load can reduce distraction. Proceedings of the National Academy of Sciences, USA. 2005; 102:16524-16529.

Minear M, Park DC. A lifespan database of adult facial stimuli. Behavior Research Methods Instruments and Computers. 2004; 36:630-633.

Mitchell KJ, Johnson MK, Raye CL, D’Esposito M. fMRI evidence of age-related hippocampal dysfunction in feature binding in working memory. Cognitive Brain Research. 2000; 10:197-206. [PubMed: 10978709]

Naveh-Benjamin M, Craik FIM. Effects of perceptual and conceptual processing on memory for words and voice: Different patterns for young and old. The Quarterly Journal of Experimental Psychology. 1996; 49(A):780-796. [PubMed: 8828404] 
Naveh-Benjamin M, Craik FI, Guez J, Dori H. Effects of divided attention on encoding and retrieval processes in human memory: Further support for an asymmetry. Journal of Experimental Psychology: Learning, Memory, and Cognition. 1998; 24(5):1091-1104.

Naveh-Benjamin M. Adult age differences in memory performance: Tests of an associative deficit hypothesis. Journal of Experimental Psychology: Learning, Memory and Cognition. 2000; 26:1170-1187.

Naveh-Benjamin M, Craik FI, Gavrilescu D, Anderson ND. Asymmetry between encoding and retrieval processes: Evidence from divided attention and a calibration analysis. Memory and Cognition. 2000; 28(6):965-976.

Naveh-Benjamin M, Hussain Z, Guez J, Bar-On M. Adult age differences in episodic memory: Further support for an associative-deficit hypothesis. Journal of Experimental Psychology: Learning, Memory and Cognition. 2003; 29(5):826-837.

Naveh-Benjamin M, Guez J, Kilb A, Reedy S. The relational memory deficit of older adults: Further support using face-name associations. Psychology and aging. 2004a; 19(3):541-546. [PubMed: 15383004]

Naveh-Benjamin M, Guez J, Shulman S. Older adults' associative deficit in episodic memory: Assessing the role of decline in attentional resources. Psychonomic Bulletin \& Review. 2004b; 11:1067-1073. [PubMed: 15875977]

Naveh-Benjamin M, Shing YL, Kilb A, Werkle-Bergber M, Lindenberger U, Li S-C. Adult age differences in memory for name-face association: The effects of intentional and incidental learning. Memory. 2009; 17(2):220-232. [PubMed: 18654927]

Navon D, Gopher D. On the economy of the human-processing system. Psychological review. 1979; 86(3):214-255.

Oh SH, Kim M-S. The role of spatial working memory in visual search efficiency. Psychonomic Bulletin \& Review. 2004; 11(2):275-281. [PubMed: 15260193]

Old SR, Naveh-Benjamin M. Memory for people and their actions: Further evidence for an age-related associative deficit. Psychology and aging. 2008; 23(2):467-72. [PubMed: 18573021]

Pilotti M, Meade ML, Gallo DA. Implicit and explicit measures of memory for perceptual information in young adults, healthy older adults, and patients with Alzheimer's disease. Experimental Aging Research. 2003; 29:15-32. [PubMed: 12735079]

Rhodes MG, Castel AD, Jacoby LL. Associative recognition of face pairs by younger and older adults: The role of familiarity-based processing. Psychology and Aging. 2008; 23(2):239-249. [PubMed: 18573000]

Troyer AK, Winocur G, Craik FI, Moscovitch M. Source memory and divided attention: reciprocal costs to primary and secondary tasks. Neuropsychology. 1999; 13(4):467-474. [PubMed: 10527055]

Troyer AK, Craik FI. The effect of divided attention on memory for items and their context. Canadian journal of experimental psychology. 2000; 54(3):161-171. [PubMed: 11021036]

Troyer AK, Hafliger A, Cadieux MJ, Craik FIM. Name and face learning in older adults: Effects of level of processing, self-generation, and intention to learn. Journals of Gerontology Series B:

Psychological Sciences and Social Sciences. 2006; 61(2):67-74.

Tulving, E. Elements of Episodic Memory. Oxford: Clarendon Press; 1983.

Woodman GF, Luck SJ. Visual search is slowed when visuospatial working memory is occupied.

Psychonomic Bulletin \& Review. 2004; 11(2):269-274. [PubMed: 15260192] 


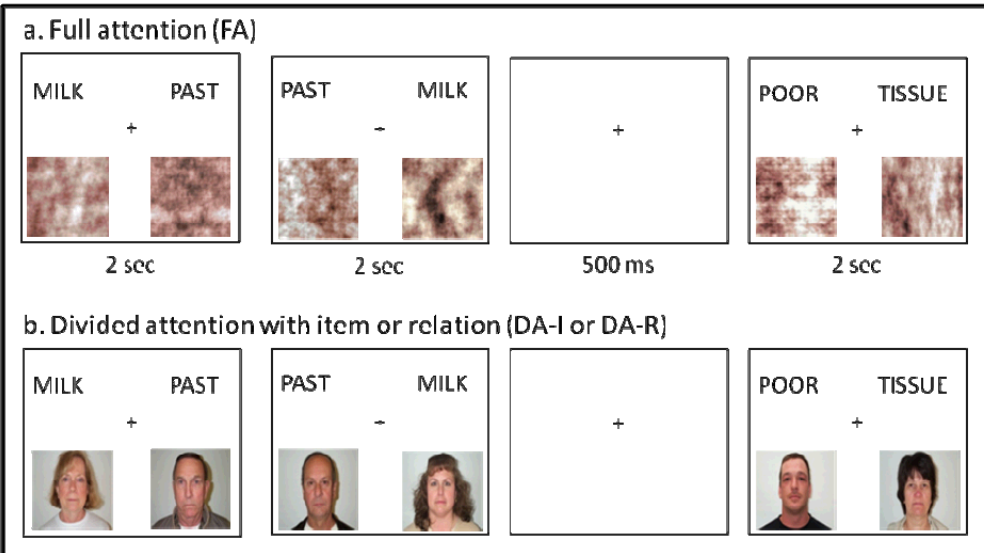

FIGURE 1.

Trial sequence for each condition in Experiment 1.

Note: In the full attention condition, participants were told to memorize both words and word pairs ignoring images presented below the word pairs (a). In the divided attention with item condition (b), participants were instructed to detect a male face in each trial while memorizing words and word pairs. In the divided attention with relation condition (b), participants were instructed to compare ages of two faces and detect an older face in each trial during encoding of words and word pairs. 


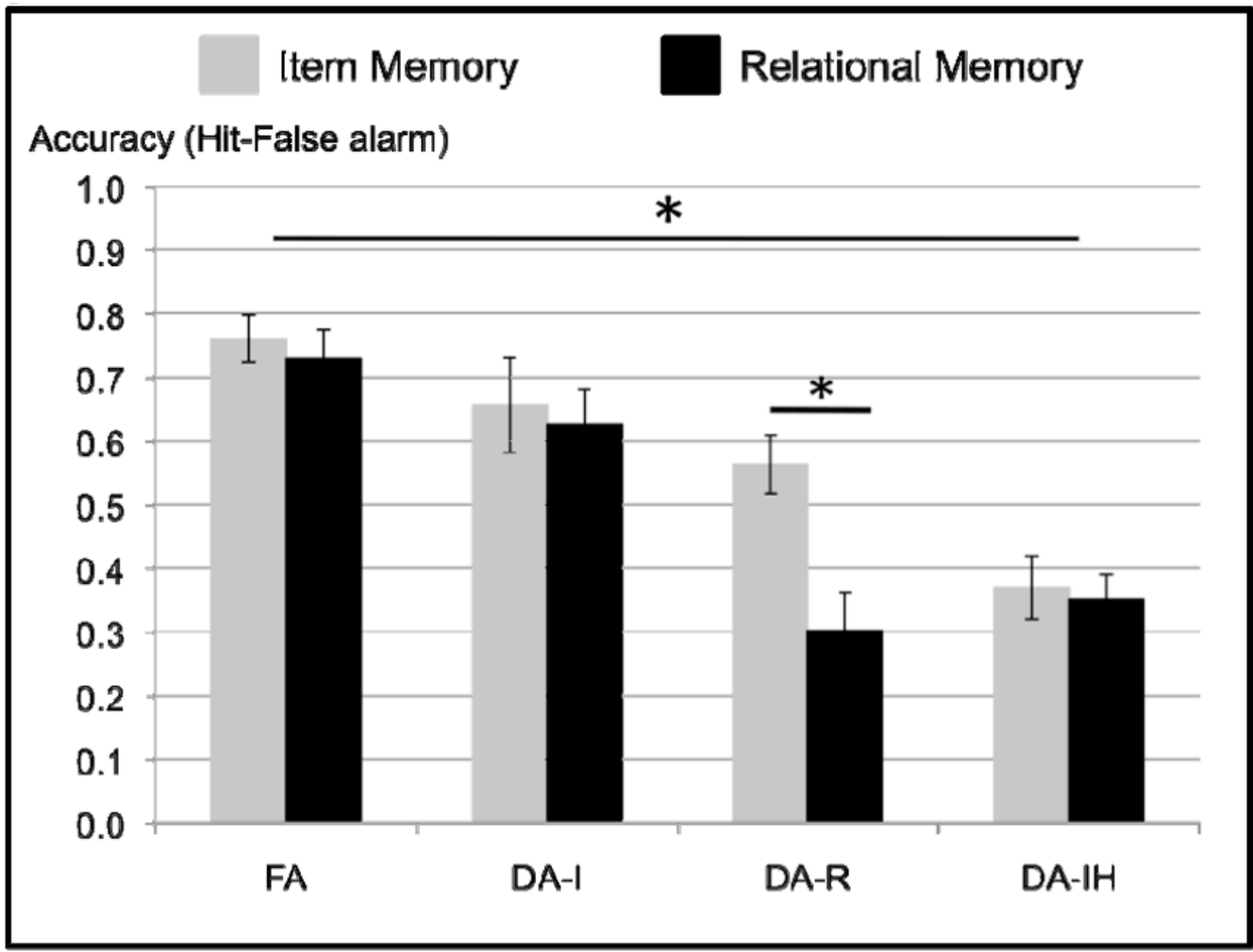

FIGURE 2.

Proportion of Hit minus Proportion of False Alarm Rates in each memory test for each group in Experiment 1.

Note: * indicates the significant effect at $\mathrm{p}<.05$. FA, Full attention; DA-I, Divided attention with item detection; DA-R, Divided attention with relation detection; DA-IH, Divided attention with item detection, harder. 


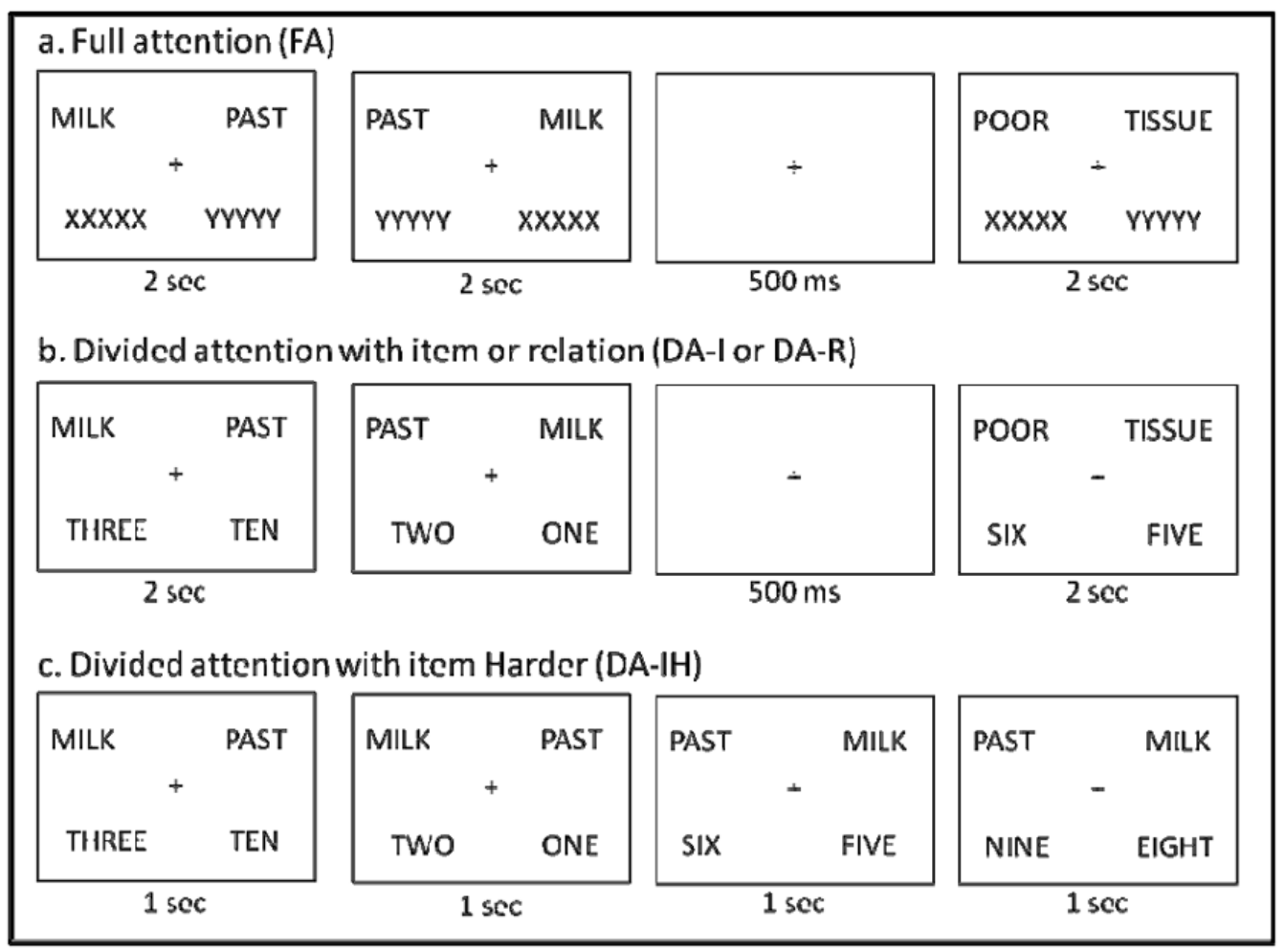

FIGURE 3.

Trial sequence for each condition in Experiment 2.

Note: In the full attention condition, participants were told to memorize both words and word pairs ignoring strings presented below the word pairs (a). In the divided attention with item condition (b), participants were instructed to detect an odd number in each trial while memorizing words and word pairs. In the divided attention with relation condition (b), participants were instructed to compare two numbers and detect a numerically bigger number in each trial during encoding of words and word pairs. In the divided attention with item, harder condition (c), participants were instructed to detect an odd number every 1 second while memorizing words and word pairs. 


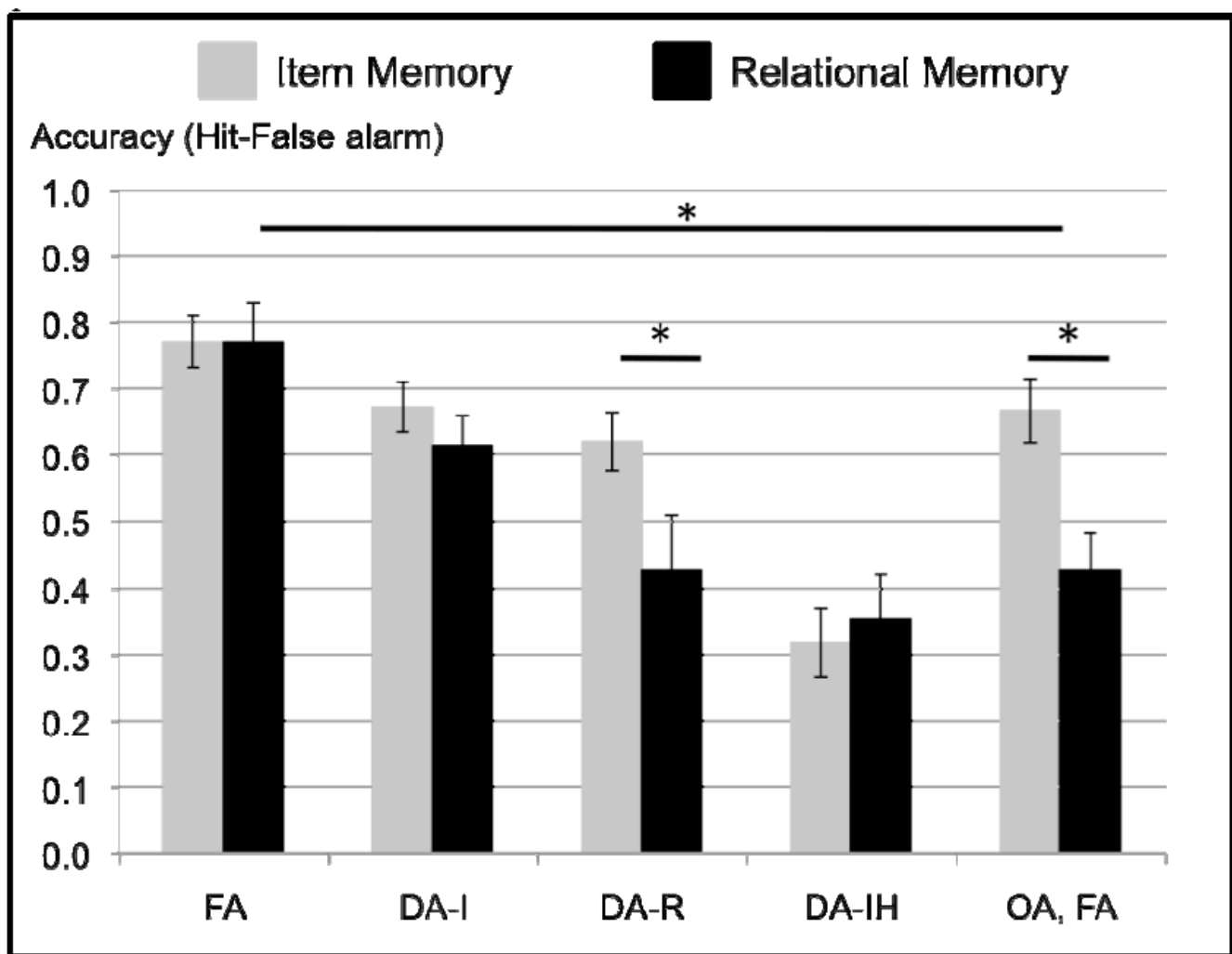

FIGURE 4.

Proportion of Hit minus Proportion of False Alarm Rates in each memory test for each group in Experiment 2.

Note: * indicates the significant effect at $\mathrm{p}<.05$. FA, Full attention; DA-I, Divided attention with item detection; DA-R, Divided attention with relation detection; DA-IH, Divided attention with item detection, harder; OA, Older adults. 


\section{Table 1}

A. Means and Standard Deviations for Accuracy and RTs (in milliseconds) in attention tasks in each of the Attention groups in Experiment 1.

\begin{tabular}{lcc}
\hline Group & Proportion Correct & RTs (ms) \\
\hline DA with Item task & $0.94(0.04)$ & $1165(205)$ \\
DA with Relational task & $0.74(0.04)$ & $1347(142)$ \\
DA with Item task, Harder & $0.75(0.14)$ & $769(41)$ \\
\hline $\begin{array}{l}\text { B. Means and Standard Deviations of memory RTs (millisecond) in the Item memory and Relational memory Tests in each of the Attention } \\
\text { groups in Experiment 1. }\end{array}$ & Item Memory & Relational Memory \\
\hline Group & $1483(316)$ & $1555(318)$ \\
\hline Full attention & $1605(221)$ & $1593(181)$ \\
DA with Item task & $1578(278)$ & $1448(254)$ \\
DA with Relational task & $1559(399)$ & $1456(271)$ \\
DA with Item task, Harder & & 1503 \\
\hline
\end{tabular}

Note: DA, Divided attention; Numbers in the parenthesis indicate standard deviation. 
Table 2

A. Means and Standard Deviations for Accuracy and RTs (in milliseconds) in attention tasks in each of the Attention groups in Experiment 2.

\begin{tabular}{lcc}
\hline Group & Proportion Correct & RTs (ms) \\
\hline YA, DA with Item task & $0.93(0.06)$ & $1354(146)$ \\
YA, DA with Relational task & $0.9(0.08)$ & $1359(183)$ \\
YA, DA with Item task, Harder & $0.68(0.1)$ & $670(48)$ \\
\hline B. Means and Standard Deviations of memory RTs (millisecond) in the Item memory and Relational memory Tests in each of the Attention \\
groups in Experiment 2. & Item Memory & Relational Memory \\
\hline Group & $1430(183)$ & $1460(222)$ \\
\hline Full attention & $1633(236)$ & $1590(270)$ \\
DA with Item task & $1540(248)$ & $1623(270)$ \\
DA with Relational task & $1435(255)$ & $1388(257)$ \\
DA with Item task, Harder & $1799(315)$ & $1995(354)$ \\
OA Full attention & & \\
\hline
\end{tabular}

Note: YA, Young adult; OA, Older adults; DA, Divided attention; Numbers in the parenthesis indicate standard deviation. 


\section{Table 3}

Means and Standard Deviations for Proportion of Hits and False Alarms Rates in the Item memory and Relational memory tests in each of the Attention groups in Experiment 2.

\begin{tabular}{lccccc}
\hline & \multicolumn{2}{c}{ Item Memory } & & \multicolumn{2}{c}{ Relational Memory } \\
\cline { 2 - 3 } Group & Hits & False Alarms & & Hits & False Alarms \\
\hline YA, Full attention & $0.78(0.14)$ & $0.01(0.04)$ & & $0.89(0.08)$ & $0.11(0.15)$ \\
YA, DA with Item task & $0.77(0.13)$ & $0.09(0.09)$ & & $0.85(0.15)$ & $0.23(0.16)$ \\
YA, DA with Relational task & $0.78(0.12)$ & $0.16(0.16)$ & & $0.74(0.16)$ & $0.31(0.17)$ \\
YA, DA with Item task, Harder & $0.57(0.12)$ & $0.25(0.15)$ & & $0.64(0.21)$ & $0.28(0.13)$ \\
OA, Full attention & $0.84(0.09)$ & $0.18(0.16)$ & & $0.79(0.11)$ & $0.37(0.19)$ \\
\hline
\end{tabular}

Note: YA, Young adult; OA, Older adults; DA, Divided attention; Numbers in the parenthesis indicate standard deviation. 